\title{
Prediction of Radiated Emissions From a Power Converter by Measuring the Common-Mode Current in the Attached Cable
}

This paper was downloaded from TechRxiv (https://www.techrxiv.org).

LICENSE

CC BY 4.0

SUBMISSION DATE / POSTED DATE

$02-12-2021 / 10-12-2021$

\section{CITATION}

ZAIKIN, DENYS; Jonasen, Stig; Mikkelsen, Simon L. (2021): Prediction of Radiated Emissions From a Power Converter by Measuring the Common-Mode Current in the Attached Cable. TechRxiv. Preprint. https://doi.org/10.36227/techrxiv.17111996.v1

DOI

10.36227/techrxiv.17111996.v1 


\title{
Prediction of Radiated Emissions From a Power Converter by Measuring the Common-Mode Current in the Attached Cable
}

\author{
Denys I. Zaikin, Member, IEEE, Stig Jonasen, and Simon L. Mikkelsen
}

\begin{abstract}
Being able to predict radiated emissions before using an accredited laboratory can be both time-effective and costeffective. This study presents a model for predicting radiated emissions from power converters by measuring the commonmode current in the attached cable. When power converters are tested for radiated emissions, the attached cables tend to be thick because of the high currents they carry. Ideally, these cables leave the chamber through connectors in an opening positioned precisely at the middle of the bottom of the turntable in keeping with CISPR 32. However, these connectors are typically not intended for currents higher than $16 \mathrm{~A}$. Consequently, such cables are usually inserted through the side wall of the chamber and are necessarily laid horizontally on the chamber floor. When the turntable is to be rotated with a device on it during a test, the length of the cable attached to the device can exceed 10 meters. The proposed model in this study is based on the transmission line model of a cable loaded with reactive impedance and the assumption that the current distribution along the cable follows a sinusoidal distribution law, much like in dipole antenna theory. The analytic equation of the radiation pattern is derived, and a simplified approximation equation has also been presented. The proposed model also works with short, attached cables and is thus universal. The Maxima software code for automated calculation of the radiated field from measurement data is supplied as supplemental material. The proposed model was experimentally validated by running the fuel cell converter module at $5 \mathrm{~kW}$ output power.
\end{abstract}

\section{INTRODUCTION}

A POWER converter must be approved for electromagnetic compatibility (EMC) on various standards, e.g., the EN 55032/CISPR 32 standard. The radiated emissions test is one of the most challenging tests to pass [1]. In such a test, the radiated noise is measured in the frequency range of $30.0 \mathrm{MHz}$ to $1.0 \mathrm{GHz}$. To save time and cost during product development, it is beneficial to be able to predict emission levels, and potentially, troubleshoot problematic radiations before using an accredited laboratory.

In power electronics, several typologies and techniques are employed to achieve low electromagnetic interference (EMI) by eliminating parasitic oscillations and ringing on printed circuit board (PCB) tracks and loops. Metal enclosures and shielding also significantly insulate noise. Hence, it can be assumed that most of the radiation is caused by the attached cable, with predominantly common-mode (CM) noise. The mechanisms underpinning this type of radiation are discussed extensively in the literature [1], [2].

Manuscript received 2021
The device under test (DUT) is placed on the turntable in a semi-anechoic chamber (SAC), and the cables are usually threaded vertically through a hole in the floor directly under the table (Fig. 1). The standard connectors on the floor are typically rated for currents less than $16 \mathrm{~A}$.

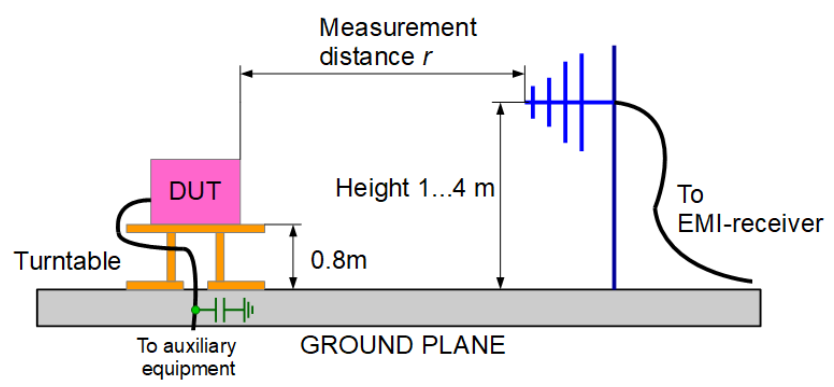

Fig. 1. DUT placement and general layout for radiated emissions tests.

Thick cables are used to test high-power converters, and the thick cable has to be threaded through the wall of the chamber, with most of the cable lying horizontally on the floor (Fig. 2). Unavoidably, the length of the cable can easily exceed $10 \mathrm{~m}$ to accommodate the rotation of the turntable. Even if the cable is threaded through a hole beneath the turntable (Fig. 2), it may still be long because the auxiliary equipment may be placed at a distance without any filtering along its length. An example of such a setup, with a long power cable lying on the floor, is shown in Fig. 3. The fuel cell converter in Fig. 3 is the subject of this research.

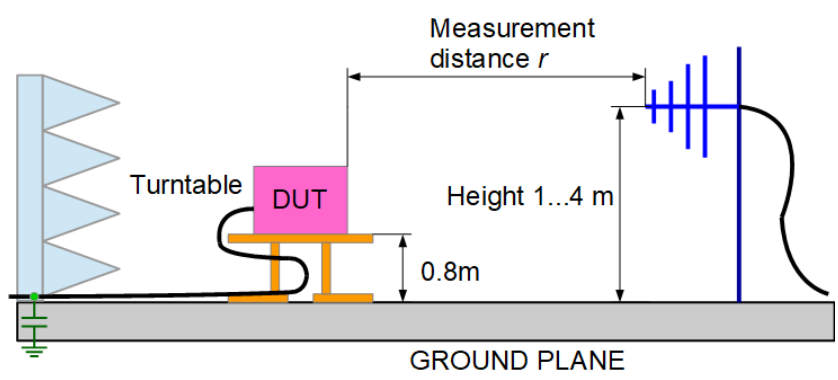

Fig. 2. DUT placement and general layout for radiated emissions tests. A long supply cable goes out through the wall of the chamber.

The crucial task is now to calculate the far-field radiation from the measured $\mathrm{CM}$ current in the attached cable. There 


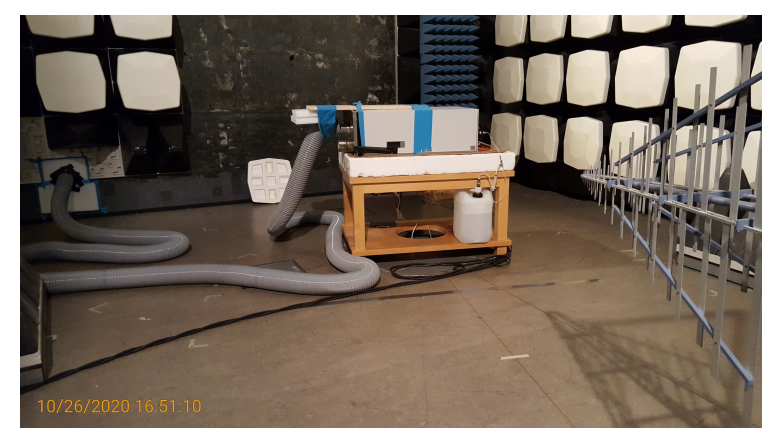

Fig. 3. Setup for radiated emissions test of a $5 \mathrm{~kW}$ fuel cell converter in an SAC chamber.

have been several studies on this problem. Several previous studies [2]-[7] utilize a model comprising a simple dipole or monopole antenna driven by a single voltage source that represents the noise source on the PCB. However, these studies assume that the input impedance is purely real and not frequency dependent. Such an approach is prone to calculation errors because the impedance of dipole antennas is generally complex and can vary considerably depending on the frequency and position of the noise source, as outlined in antenna theory [8]-[10]. Furthermore, these methods are dependent on the noise voltage, which is impossible to predict in real complex PCBs, and modern power converters typically contain many noise sources distributed across the entire PCB.

In their study, Jia et al. [11] use precise CM current measurements along the cable to calculate the radiated field. To fit the measured current amplitude, 100 points were required for magnitude measurement and recovery of phase information using an optimization procedure based on a trust-regionreflective method and a multi-start point algorithm. Evidently, this approach requires many points to measure the current along the cable and employs a complex numerical calculation. Furthermore, it has been reported that the method cannot be used in the low frequency range around $30 \mathrm{MHz}$, within which the algorithm fails. These disadvantages limit its practical implementation in power electronics with long cables attached.

In a study by McCormick [12], an artificial network is used to predict the radiated pattern by analyzing results from previous reference measurements and a numerical simulation. This method requires several commercial software (SW), and the artificial network uses only PCBs with a rectangle-shaped base learn. Thus, this method may be expensive and time consuming.

A power electronics converter typically has peaks in radiation within the low frequency range of 30-300 MHz. These peaks correspond to the cable resonances. The goal of this study is to derive a model and an equation with which to calculate the radiated far field from the previously measured $\mathrm{CM}$ current in the cable attached. The closed form equations are successfully derived, and a Maxima software file for automating the calculation is provided for users to troubleshoot radiated emissions issues efficiently.

In the subsequent sections, we describe the process of developing the proposed model. In Section (II), we describe

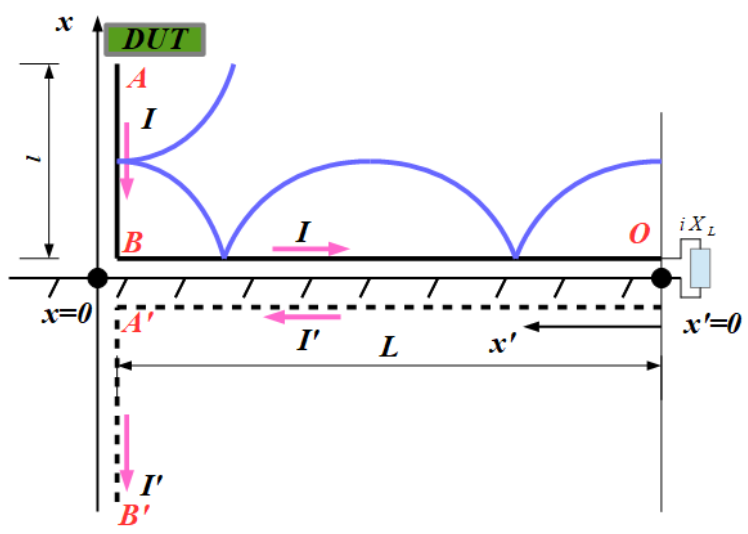

Fig. 4. Transmission line model representation of the cable attached to the DUT.

the model based on a transmission line model. The derivation of the main equation is presented in Section (III), while a simplified equation for a configuration with a long cable is presented in Section (IV). A configuration with a short cable is considered in Section (V), and experimental validation of the proposed model is presented in Section (VII).

\section{DESCRIPTION OF THE MODEL}

A model of the cable connected to a DUT can be developed from the original setup in the SAC shown in Fig. 1. The proposed model consists of a transmission line (TL), which represents the cable attached to the PCB or enclosure, as shown in Fig. 4. The portion of the cable placed on the floor of the chamber has a length of $L$ and the vertical portion of the cable has a length of $l$. The $x^{\prime}$ axis begins at the end of the cable and is oriented horizontally toward the DUT. It is assumed that the TL has a purely reactive impedance at its end (at the coordinate $x^{\prime}=0$ ). This assumption guarantees a voltage standing wave ratio $(V S W R) \gg 1$, such that the peak current in the cable is maximized for the worst case analysis. The purely reactive impedance $Z_{L}=i X_{L}$ at the end of the TL represents the connection to the load, the noise filter, or tight coupling to the ground plane at high frequency (HF). The $x$ axis begins at point $x^{\prime}=L$ and is oriented vertically upward. The cable is connected to the DUT at the coordinate $x=l$. Thus, the $x$ axis is approximately a continuation of the $x^{\prime}$ axis when the cable changes its orientation. The current $I$ in the TL and its direction are also indicated. Because the SAC chamber has a metal floor, there is an image plane effect, which causes an imaginary current $I^{\prime}$ to flow in the mirrored semi-space beneath the floor. This imaginary current $I^{\prime}$ is highlighted in Fig. 4 with a dashed line. These currents, $I$ and $I^{\prime}$, in the vertical portion of the cable are used to calculate the radiated field in Section (III).

Here, we make the following assumption: the current distribution along the cable follows a sinusoidal distribution law, much like in symmetric dipole antenna theory [8]-[10]. A simulation based on the moment method (using the MMANAGAL simulation software) proves that this assumption is very close to reality (Fig. 5). The other assumptions are that the 

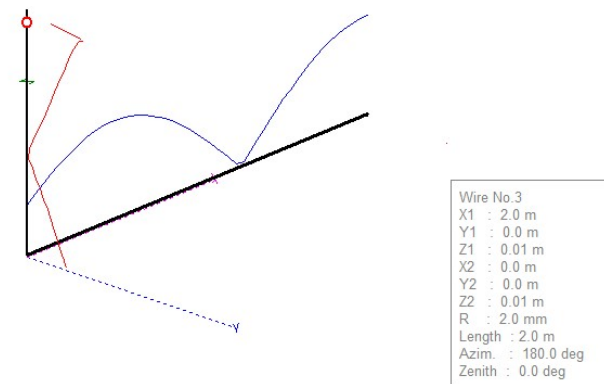

Fig. 5. Simulation of current distribution along the cable using the MMANA-GAL software and the method-of-moments.

TL is lossless and that the vertical and horizontal parts of the cable have the same characteristics as a TL with a wave number $k=2 \pi / \lambda$, and a characteristic impedance $Z_{c}$, where $\lambda$ is the wavelength in the cable.

A summary of the assumptions can be listed as follows:

- The cable attached to the DUT can be represented as a TL with purely reactive impedance at its end.

- The current distribution along the cable follows a sinusoidal distribution law.

- The cable is modeled on the lossless TL model.

- The wavelength, wave number, and characteristic impedance are the same for the vertical and horizontal portions of the TL.

- The wave number $k$ and the speed of light $c$ are the same for the cable and in free space.

- Because of the image plane effect, the horizontal portion of the cable radiates neglected energy, and thus, can be ignored in calculating the radiated field.

\section{DERIVATION OF EQUATIONS}

\section{A. Current in the transmission line}

First, the current in the TL loaded with purely reactive impedance $Z_{L}=i X_{L}$ at its end (Fig. 4) can be expressed as follows

$$
\dot{I}\left(x^{\prime}\right)=\dot{I}_{m} \cos \left(k x^{\prime}+\phi_{L}\right)
$$

where $k=2 \pi / \lambda$ is a wave number, $\dot{I}\left(x^{\prime}\right)$ is the complex current in the cable at the coordinate $x^{\prime}$, and $\dot{I}_{m}$ is the complex amplitude of the current (which must be determined to calculate the radiated field) in the cable. Phase $\phi_{L}$ depends on the TL load and is equal to

$$
\phi_{L}=\arctan \left(\frac{X_{L}}{Z_{c}}\right)
$$

The following scenarios can be considered:

- The TL is shorted at its end or loaded with a large capacitance, e.g., a П-type EMI filter capacitor, and $X_{L}=0$ and $\phi_{L}=0$.

- The TL is loaded with a large inductance, e.g., with an $T$ type EMI filter inductor or with ferrite beads, and $X_{L}=$ $+\infty$ and $\phi_{L}=+\pi / 2$.

- The TL is open at its end or loaded with a small capacitance, and $X_{L}=-\infty$ and $\phi_{L}=-\pi / 2$.
At this point, it is necessary to express the current in relation to the $x$ axis, which represents a continuation of the $x^{\prime}$ axis in the vertical direction. Thus, Eq. (1) can be rewritten as follows

$$
\dot{I}(x)=\dot{I}_{m} \cos \left(k(x-L)+\phi_{L}\right) .
$$

Then, because the image plane effect from the current in the imaging portion of the cable $A^{\prime} B^{\prime}$ under the plane has the same direction and value as in the portion of the cable $A B$ above the plane, Eq. (3) can be further modified to a universal form with positive and negative coordinates

$$
\dot{I}(x)=\dot{I}_{m} \cos \left(k(|x|-L)+\phi_{L}\right),-l \leq x \leq l
$$

or

$$
\begin{aligned}
& \dot{I}(x)=\dot{I}_{m} \cos \left(k(x-L)+\phi_{L}\right), 0 \leq x \leq l, \\
& \dot{I}(x)=\dot{I}_{m} \cos \left(k(x+L)-\phi_{L}\right),-l \leq x \leq 0 .
\end{aligned}
$$

\section{B. Radiation pattern of the cable}

The far-field radiation from the vertical portion of the cable can be represented as a superposition of the far fields of elementary electric dipoles, as illustrated in Fig. 6.

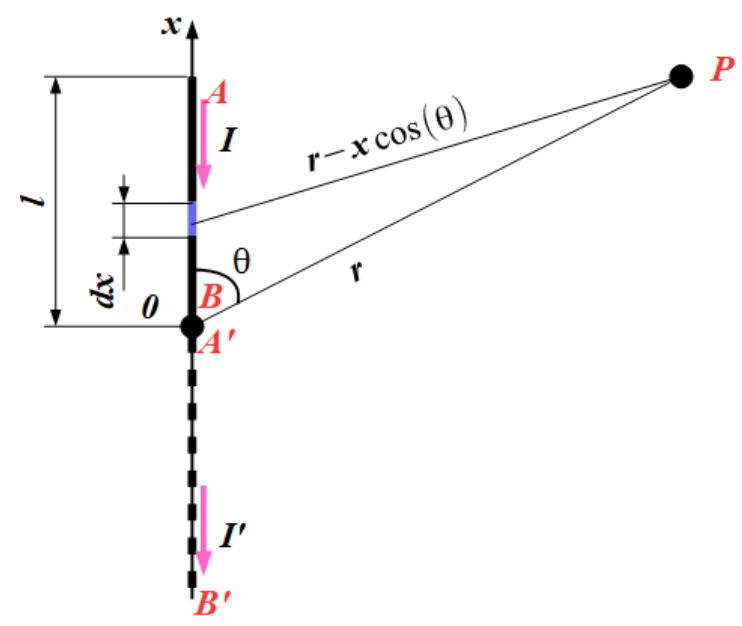

Fig. 6. Diagram of a radiated field calculated as a superposition of electric dipole radiation.

The far zone electric field $E_{\theta \text { dipole }}$ of one elementary electric dipole of length $d l$ can be expressed as [8]-[10]

$$
\dot{E}_{\theta \text { dipole }}=\frac{i 60 \pi \dot{I} d l}{\lambda} \frac{e^{-i k r}}{r} \sin \theta,
$$

where $i=\sqrt{-1}, k=2 \pi / \lambda$ is a wave number, and $\lambda$ is the wavelength in free space. $r$ is the distance from the observation point $P$, and in the far zone, $r \gg l^{2} / \lambda$ [9]. Considering Fig. 6, the contribution of the cable element $d x$ to the electric field can be derived as

$$
d \dot{E}_{\theta}=\frac{i 60 \pi \dot{I}(x) d x}{\lambda} \frac{e^{-i k r}}{r} \sin \theta,
$$

or, substituting in Eq. (4), the following expression can be derived:

$$
\begin{aligned}
d \dot{E}_{\theta}=\frac{i 60 \pi \dot{I}_{m}}{\lambda} & \frac{e^{-i k r}}{r} \\
& \times \sin \theta e^{i k x \cos \theta} \cos \left(k(|x|-L)+\phi_{L}\right) d x,
\end{aligned}
$$


Eq. (8) can be rewritten in a simplified form, which is easier to integrate along the $x$ axis, as follows

$$
d \dot{E}_{\theta}=\frac{i 60 \pi \dot{I}_{m}}{\lambda} \frac{e^{-i k r}}{r} d F(\theta, x)
$$

where

$$
d F(\theta, x)=\sin \theta e^{i k x \cos \theta} \cos \left(k(|x|-L)+\phi_{L}\right) d x .
$$

To determine the total electric field at point $P$, Eq. (9) must be integrated along the $x$ axis with limits $-l \leq x \leq l$

$$
\dot{E}_{\theta}(P)=\frac{i 60 \pi \dot{I}_{m}}{\lambda} \frac{e^{-i k r}}{r} F(\theta, x) .
$$

Thus, the radiation pattern system factor $F(\theta, x)$ can be determined by integrating Eq. (10)

$$
\begin{aligned}
& F(\theta, x)= \\
& \sin \theta \int_{-l}^{l} e^{i k x \cos \theta} \cos \left(k(|x|-L)+\phi_{L}\right) d x,
\end{aligned}
$$

or, considering Eq. (5)

$$
\begin{aligned}
F(\theta, x)= & \\
\sin \theta & \left(\int_{-l}^{0} e^{i k x \cos \theta} \cos \left(k(x+L)-\phi_{L}\right) d x\right. \\
& \left.+\int_{0}^{l} e^{i k x \cos \theta} \cos \left(k(x-L)+\phi_{L}\right) d x\right) .
\end{aligned}
$$

After the preceding calculation, the following equation can be derived:

$$
\begin{aligned}
F(\theta, x)= & -\frac{2}{k \sin \theta} \\
\times & \left(\cos \left(k(l-L)+\phi_{L}\right) \cos \theta \sin (k l \cos \theta)\right. \\
& -\sin \left(k(l-L)+\phi_{L}\right) \cos (k l \cos \theta) \\
& \left.-\sin \left(L k-\phi_{L}\right)\right) .
\end{aligned}
$$

Substituting Eq. (14) into Eq. (11) will yield the expression for the total field

$$
\begin{aligned}
\dot{E}_{\theta}(\theta, r)= & -\frac{i 60 \dot{I}_{m}}{r} \frac{e^{-i k r}}{\sin \theta} \\
\times & \left(\cos \left(k(l-L)+\phi_{L}\right) \cos \theta \sin (k l \cos \theta)\right. \\
& -\sin \left(k(l-L)+\phi_{L}\right) \cos (k l \cos \theta) \\
& \left.-\sin \left(L k-\phi_{L}\right)\right) .
\end{aligned}
$$

Eq. (15) can be used to directly calculate the electric field in the far zone. This equation accounts for all lengths $L$ and can thus be applied to all cable configurations. However, from Eq. (15), it is evident that for every frequency $f$ (i.e., for every $k=2 \pi / \lambda=2 \pi f / c$ ) the angle $\theta$ must be numerically calculated or scanned to determine the maximum field strength for use in the worst case analysis.

Another drawback of using Eq. (15) directly is that the final shape of the field strength derived from the frequency will have many zeros and local maxima due to the cable resonances (Fig. 7). In practice, one of these zeros in the field strength characteristics may overlap with a real problematic frequency, such that the prediction indicates a low level of radiation.

In conclusion, it is desirable to obtain the envelope of the transfer characteristic expressed in Eq. (15) in order to determine the maximum possible field strength for which the expression does not depend on the angle $\theta$ and the parameter $L$. Eq. (15) is too complex to be solved using classical algebra methods because the function comprises an angle $\theta$ in phase and magnitude members, and is thus transcendental. In the next section, we demonstrate the derivation of approximate envelopes for two scenarios: for the long portion of the cable, $L \gg l$; and for the short horizontal portion of the cable, $L \ll l$. It is desirable that the final approximated equations are based on physical sense.

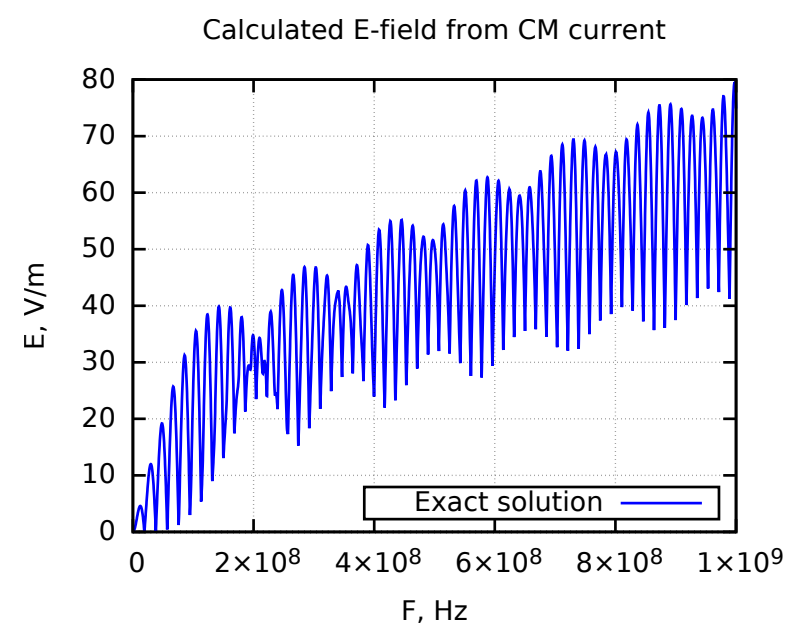

Fig. 7. Example of field calculation using Eq. (15). For every frequency, the angle $\theta$ was numerically calculated for the maximum field strength. $l=1.0 \mathrm{~m}, L=8.4 \mathrm{~m}, I_{m}=1 \mathrm{~A}$, $r=3 m, \phi_{L}=\pi / 2$.

\section{Simplified EQUATION FOR A LONG CABLE CONFIGURATION}

\section{A. Long cable configuration: Frequency range $f \leq c /(2 l)$}

The entire frequency range can be split into two sections. As established in antenna theory [8]-[10], if the length of the symmetric dipole antenna is less than the wavelength $2 l \leq \lambda$, then the maximum strength of the radiated field is always located at $\theta=\pi / 2$. In this case, Eq. (15) can be rewritten for a scenario where $f \leq c /(2 l)$, as

$$
\begin{aligned}
\left.\dot{E}_{\theta}(r)\right|_{f \leq c /(2 l)} & =\frac{i 60 \dot{I}_{m}}{r} e^{-i k r} \\
\times & \left(2 \cos \left(k(l / 2-L)+\phi_{L}\right) \sin (k l / 2)\right) .
\end{aligned}
$$

Thus, the maximum magnitude of the electric field of a long cable $L \gg l$ at frequencies $f \leq c /(2 l)$ can now be expressed 
by calculating the absolute value of Eq. (16)

$$
\begin{aligned}
&\left.E_{\theta}^{\max }(r)\right|_{f \leq c /(2 l)}=\frac{60 I_{m}}{r} \\
& \times 2 \cos \left(k(l / 2-L)+\phi_{L}\right) \sin (k l / 2) .
\end{aligned}
$$

Analyzing Eq. (17), it is apparent that when $L \gg l$, the member $\sin (k l / 2)$ is an approximate envelope for the member $\cos \left(k(l / 2-L)+\phi_{L}\right)$, with the wave number $k$ considered as a variable. Thus, the envelope can be expressed, with the exclusion of the parameter $L$, as

$$
\left.E_{\theta}^{\max }(f)\right|_{f \leq c /(2 l)} ^{L \gg l}=\frac{60 I_{m}(f)}{r}(2 \sin (\pi f l / c)) .
$$

B. Long cable configuration: Frequency range $f \leq c /(2 l)$, with measurement only in the vertical portion of the cable

It should be noted that in Eq. (18), the radiated field is calculated using a measured maximum magnitude of the current in the current node $I_{m}$ somewhere in the cable. For the low frequency range $f \leq c /(2 l)$, the current node might not be located in the vertical portion of the cable but somewhere in the horizontal portion, as follows from TL theory. Thus, for the low frequency range $f \leq c /(2 l)$, measurement of the maximum current magnitude must also be performed along the horizontal portion of the cable. For higher frequencies $f>c /(2 l)$, the current node can always be found in the vertical portion of the cable, and making this measurement along the horizontal portion can be skipped.

To avoid having to make the measurement along the horizontal portion of the cable, the following approach can be considered: the magnitude $I_{m}$ can be estimated by measuring the maximum $\mathrm{CM}$ current $I_{m}^{\prime}$ in the vertical portion of the cable (Fig. 8).

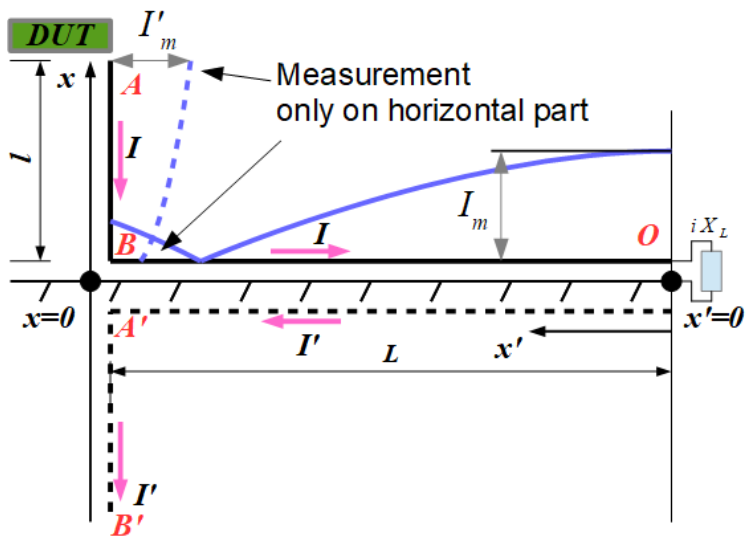

Fig. 8. Transmission line model representing the cable attached to the DUT for a low frequency configuration $f \leq c /(2 l)$.

Using Eq. (5), the magnitude $I_{m}$ can be calculated by determining the maximum magnitude in the vertical segment $I_{m}^{\prime}(x)$ at some point with coordinate $x$, as follows

$$
I_{m}=\frac{I_{m}^{\prime}(x)}{\cos \left(k(x-L)+\phi_{L}\right)}, 0 \leq x \leq l .
$$

To use Eq. (19), the parameters $L$ and $k$ must be included. Based on the assumptions made in Section II, it is impossible to precisely calculate the magnitude $I_{m}$ because of the tolerance of these parameters. It is desirable to have a calculation that is independent of parameter $L$. A worst case analysis can be implemented. In this case, we can estimate the minimal value of the maximum magnitude, $I_{m}^{\prime}$, that yields the maximum correction coefficient, $\frac{I_{m}^{\prime}}{I_{m}}$, for Eq. (18). Hence, such an approach will result in a potential overestimation of the radiated field. The expression $\dot{I}(x)=\dot{I}_{m} \cos \left(k(x-L)+\phi_{L}\right)$ can be represented on the complex variable plane as a vector diagram (Fig. 9a). It is apparent that we can determine the

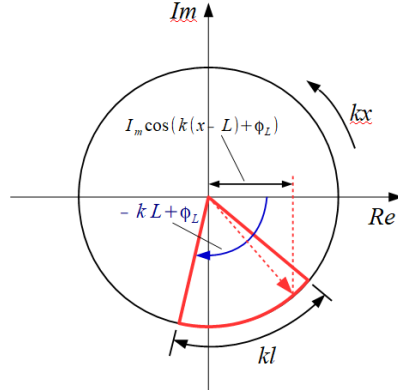

(a)

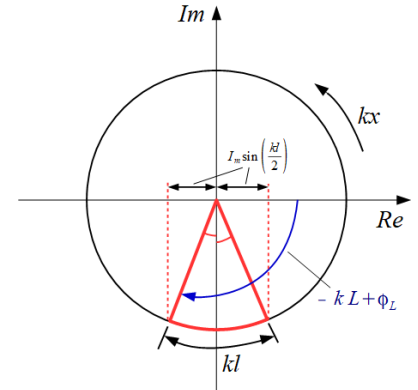

(b)
Fig. 9. Vector diagram of equation $\dot{I}(x)=$ $\dot{I}_{m} \cos \left(k(x-L)+\phi_{L}\right)$.(a) Common case, (b) Worst case for $I_{m}^{\prime}$ estimation.

worst case, which is when the maximum magnitude $I_{m}^{\prime}$ has a minimal value. This corresponds to the symmetrical placement of the vertical segment of the cable $(k l)$ in relation to an imaginary axis $I m$ in a complex plane (Fig. 9b). As can be seen, the correction coefficient will be equal to $\frac{I_{m}^{\prime}}{I_{m}}=$ $\sin (k l / 2)=\sin (\pi f l / c)$. Eq. (18) can now be modified to use the $I_{m}^{\prime}$ measurement instead of $I_{m}$

$$
\left.E_{\theta}^{\max }(f)\right|_{f \leq c /(2 l)} ^{L \gg l}=2 \frac{60 I_{m}^{\prime}(f)}{r} .
$$

The drawback of using Eq. (20) instead of Eq. (18) and measuring the CM current only in the vertical portion of the cable is that Eq. (20) yields an overestimation of the radiated field, which might be acceptable because an overestimation is preferable to an underestimation when preparing for accredited tests. On the other hand, Eq. (20) has a simple form and speeds up EMC troubleshooting by circumventing the scanning of long cables for the maximum magnitude.

\section{Long cable configuration: Frequency range $f \geq c /(2 l)$}

In the case of frequencies higher than $f>c /(2 l)$, and considering condition $L \gg l$, Eq. (15) can be rewritten as follows

$$
\begin{aligned}
& \dot{E}_{\theta}(\theta, r)=-\frac{i 60 \dot{I}_{m}}{r} \frac{e^{-i k r}}{\sin (\theta)} \\
& \quad \times\left(A \cos \left(k(l-L)+\phi_{L}+\phi\right)-\sin \left(L k-\phi_{L}\right)\right)
\end{aligned}
$$


where

$$
A=\sqrt{\cos ^{2}(k l \cos \theta) \sin ^{2} \theta+\cos ^{2} \theta}
$$

is a magnitude and $\phi$ is some phase. This phase member depends on $k l$, and thus oscillates more slowly than the $k(l-L)$ member, considering the wave number $k$ as a variable. It can now be assumed that the maximum magnitude of the electric field (Eq. (21)) can be achieved when the magnitude $A$ (Eq. (22)) is maximized $A=1$. This condition can be attained when the following equation is solved $\cos ^{2}(k l \cos (\theta))=1$, or rewritten as $\cos ^{2}\left(\frac{2 \pi f l}{c} \cos (\theta)\right)=1$. On determining the solution, it is possible to derive

$$
\cos (\theta)=\frac{n}{\frac{2 f l}{c}}, n=0, \pm 1, \pm 2 \ldots
$$

In Eq. (23), the number $n$ should be chosen such that the $\sin \theta$, and thus, the angle $\theta$, are minimal, as can be seen in Eq. (21). In other words, the $\cos \theta$ chosen must be as close as possible to 1 . Thus, the number $n$ can be selected such that it follows and is as close as possible to the $\frac{2 f l}{c}$ value, e.g., equal to the largest integer less than or equal to $\frac{2 f l}{c}$. Finally, to have a smooth characteristic, Eq. (23) can be transformed to

$$
\cos \theta=\frac{\frac{2 f l}{c}-1}{\frac{2 f l}{c}}=1-\frac{c}{2 f l} .
$$

Furthermore, to maximize Eq. (21), it should be assumed that $\sin \left(k L-\phi_{L}\right)=-1$. Thus, the maximum magnitude of the electric field of the long cable $L \gg l$ at frequencies $f>c /(2 l)$ can now be expressed (after calculating the absolute value of Eq. (21)) as

$$
\left.E_{\theta}^{\max }(f)\right|_{f>c /(2 l)} ^{L \gg l}=\frac{60 I_{m}(f)}{r} \frac{2}{\sqrt{1-\left(1-\frac{c}{2 f l}\right)^{2}}} .
$$

Eq. (18) and (25) can now be combined to derive a closed form equation for the long cable $L \gg l$ configuration

$$
\left.E_{\theta}^{\max }(f)\right|_{L \gg l}= \begin{cases}\frac{60 I_{m}(f)}{\frac{60 I_{m}(f)}{r}} \frac{\left(2 \sin \left(\frac{\pi f l}{c}\right)\right),}{\sqrt{1-\left(1-\frac{c}{2 f l}\right)^{2}},} & \text { if } f \leq \frac{c}{2 l} \\ \frac{2}{2 l}\end{cases}
$$

Eq. (26) represents an envelope approximation of Eq. (15). A comparison of the approximation equation and the full equation is presented in Fig. 10. It is apparent that the approximation corresponds to the actual characteristics with good accuracy.

Eq. (26) must be used when the maximum magnitude $I_{m}$ is scanned along the entire cable length, including the horizontal portion. If the measurement is performed only along the vertical portion of the cable, and a magnitude $I_{m}^{\prime}$ is obtained, Eq. (26) should be replaced with the following (considering Eq. (20))

$$
\left.E_{\theta}^{\max }(f)\right|_{L \gg l}= \begin{cases}2 \frac{60 I_{m}^{\prime}(f)}{r}, & \text { if } f \leq \frac{c}{2 l} \\ \frac{60 I_{m}^{\prime}(f)}{r} \frac{2}{\sqrt{1-\left(1-\frac{c}{2 f l}\right)^{2}}}, & \text { if } f>\frac{c}{2 l}\end{cases}
$$

A comparison of the transfer coefficients from Eq. (26) and Eq. (27) is presented in Fig. 11.

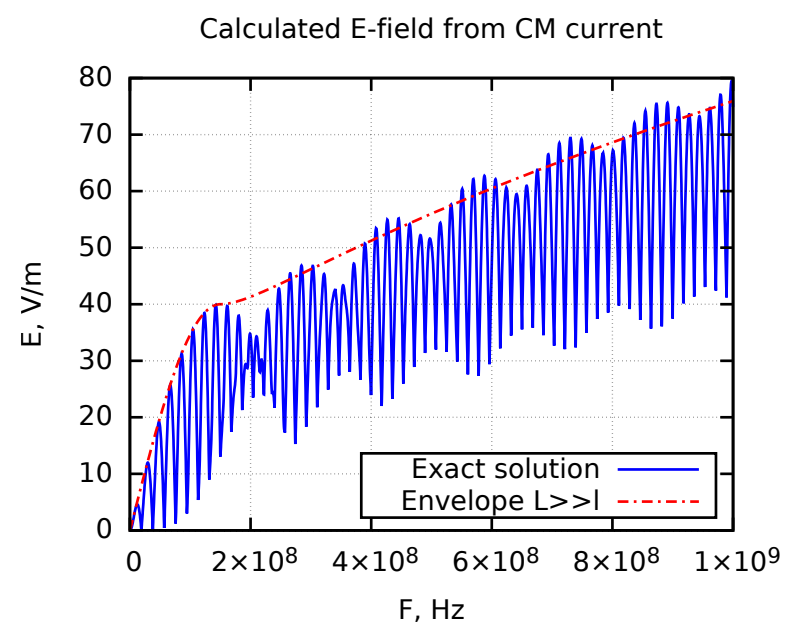

Fig. 10. Comparison of the exact solution (Eq. (15)) and the approximation equation for $L \gg l$ (Eq. (26)). $l=1.0 \mathrm{~m}$, $\boldsymbol{L}=\mathbf{8 . 4} \boldsymbol{m}, I_{m}=1 A, r=3 m, \phi_{L}=\pi / 2$.

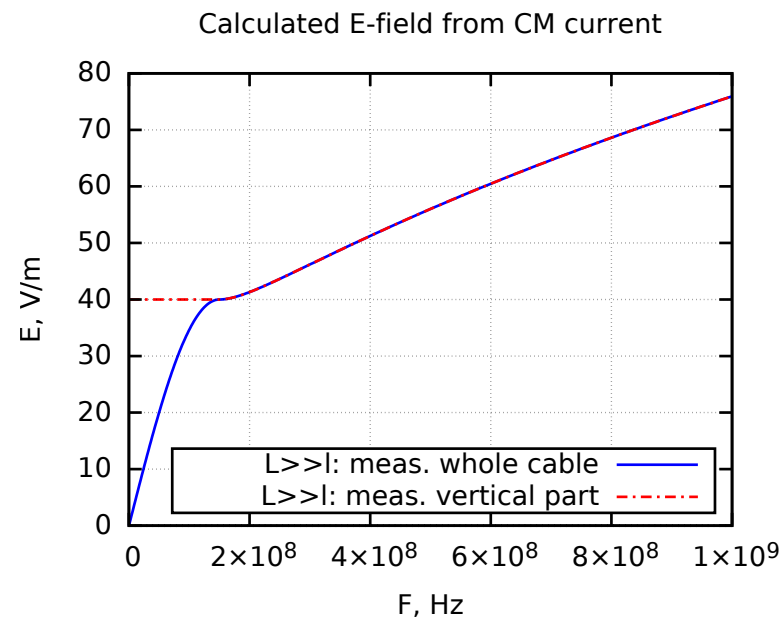

Fig. 11. Comparison of transfer coefficients from Eq. (26) and Eq. (27) for the approximation equation for $L \gg l . l=1.0 \mathrm{~m}$, $\boldsymbol{L}=\mathbf{8 . 4} \boldsymbol{m}, I_{m}=1 A, r=3 \mathrm{~m}$.

\section{Simplified EQUATION FOR A SHORT CABLE CONFIGURATION}

For a short cable $L \ll l$, it can be assumed that $L=0$, i.e., in an extreme case, the cable does not have a horizontal portion. In this scenario, Eq. (15) can be transformed as follows

$$
\begin{aligned}
\left.\dot{E}_{\theta}(\theta, r)\right|_{L \ll l} & =-\frac{i 60 \dot{I}_{m}}{r} \frac{e^{-i k r}}{\sin \theta} \\
& \times\left(\cos \left(k l+\phi_{L}\right) \cos \theta \sin (k l \cos \theta)\right. \\
& \left.-\sin \left(k l+\phi_{L}\right) \cos (k l \cos \theta)+\sin \left(\phi_{L}\right)\right) .
\end{aligned}
$$


A. Short cable configuration: Frequency range $f \leq c /(2 l)$

As previously mentioned in Section IV, the entire frequency range can be split into two divisions. If the length of the symmetric dipole antenna is less than the wavelength $2 l \leq \lambda$, then the maximum strength of the radiated field is always located at $\theta=\pi / 2,[8]-[10]$. In this scenario, Eq. (28) can be rewritten for the scenario where $f \leq c /(2 l)$

$$
\left.E_{\theta}^{\max }(r)\right|_{L \ll l} ^{f \leq c /(2 l)}=\frac{60 I_{m}}{r}\left(\sin \left(\phi_{L}\right)-\sin \left(k l+\phi_{L}\right)\right) .
$$

which can be rewritten as follows

$$
\begin{aligned}
\left.E_{\theta}^{\max }(r)\right|_{L \ll l} ^{f \leq c /(2 l)} & =\frac{60 I_{m}}{r} \\
& \times\left(2 \sin (-k l / 2) \cos \left(k l / 2+\phi_{L}\right)\right) .
\end{aligned}
$$

It is apparent that the magnitude of Eq. (30) is maximal when $\phi_{L}= \pm \pi / 2$. Finally, in terms of frequency, Eq. (30) can be rewritten as

$$
\left.E_{\theta}^{\max }(f)\right|_{L \ll l} ^{f \leq c /(2 l)}=\frac{60 I_{m}(f)}{r}\left(2 \sin ^{2}(\pi f l / c)\right) .
$$

\section{B. Short cable configuration: Frequency range $f \geq c /(2 l)$}

Notably, the approach used in Section IV for Eq. (21) cannot be employed anymore because the amplitude $A$ and phase $\phi$ will oscillate with a period of a similar order.

From Section V-A, it is assumed that $\phi_{L}=\pi / 2$. Thus, Eq. (28) can now be rewritten as follows

$$
\begin{aligned}
\left.\dot{E}_{\theta}(\theta, r)\right|_{L \ll l} & =-\frac{i 60 \dot{I}_{m}}{r} \frac{e^{-i k r}}{\sin \theta} \\
& \times(\sin (k l) \cos \theta \sin (k l \cos \theta) \\
& +\cos (k l) \cos (k l \cos \theta)+\sin (\pi / 2)) .
\end{aligned}
$$

Using some algebra transformations, it is possible to derive

$$
\begin{aligned}
\left.\dot{E}_{\theta}(\theta, r)\right|_{L \ll l} & =-\frac{i 60 \dot{I}_{m}}{r} \frac{e^{-i k r}}{\sin \theta} \\
\times \frac{1}{2}(\cos (k l(1+\cos \theta))(1-\cos \theta) & \\
& +\cos (k l(1-\cos \theta)(1+\cos \theta)+2) .
\end{aligned}
$$

It is apparent that Eq. (33) consists of an expression in brackets that can be represented as the sum of two oscillations, considering the wave number $k$ as a variable. One oscillation has a magnitude $A_{1}=1-\cos \theta$ and a phase $\Phi_{1}=k l(1+\cos \theta)$, and the other oscillation has a magnitude $A_{2}=1+\cos \theta$ and a phase $\Phi_{2}=k l(1-\cos \theta)$. To have maximum radiation, these two oscillations must interfere in phase and be positive due to the presence of the +2 member in the brackets. Thus, the sum of these oscillations will have a peak value equal to $A_{1}+A_{2}=2$. Now, it is necessary to determine a condition for the oscillations to be summarized in phase, as follows

$$
k l(1+\cos \theta)=\pi a, a=0,2,4, \ldots
$$

$$
k l(1-\cos \theta)=\pi b, b=0,2,4, \ldots
$$

The parameters $a$ and $b$ must be positive even numbers because the expressions $k l(1-\cos \theta)$ and $k l(1+\cos \theta)$ cannot be negative from a physical point of view. Taking the sum of Eq. (34) and Eq. (35), the following expression for $k l$ can be derived

$$
k l=\pi(a+b) / 2=\pi m, m=0,1,2, \ldots,
$$

where $m$ is some non-negative number.

Subtracting Eq. (35) from Eq. (34), $\cos (\theta)$ can be expressed as follows

$$
\cos \theta=\frac{\pi(a-b)}{2 k l}=\frac{\pi n}{k l}, n=0,1,2, \ldots .
$$

Considering Eq. (36), the following expression can be derived

$$
\cos \theta=\frac{\pi(a-b)}{2 k l}=\frac{\pi n}{\pi m}=\frac{n}{m} .
$$

where $n=1,2,3, \ldots$ and $m=1,2,3, \ldots$ Again, the numbers $n$ and $m$ should be chosen such that the $\sin \theta$, and thus angle $\theta$, are minimal (Eq. (28)). In other words, the $\cos (\theta)$ chosen must be as close as possible to 1 . In this case, the number $n$ should follow and should be as close as possible to the number $m$, and thus $n=m-1$. Now, the following expression can be derived

$$
\cos \theta=\frac{m-1}{m} .
$$

Considering that $\pi m=k l$ (Eq. (36)), Eq. (39) can be transformed as follows

$$
\cos \theta=\frac{\frac{k l}{\pi}-1}{\frac{k l}{\pi}} .
$$

Finally, we obtain

$$
\cos \theta=\frac{\frac{2 f l}{c}-1}{\frac{2 f l}{c}}=1-\frac{c}{2 f l} .
$$

Thus, the maximum magnitude of the electric field of the short cable $L \ll l$ at frequencies $f>c /(2 l)$ can now be expressed (after calculating the absolute value of Eq. (33))as

$$
\left.E_{\theta}^{\max }(f)\right|_{L \ll l} ^{f>c /(2 l)}=\frac{60 I_{m}(f)}{r} \frac{2}{\sqrt{1-\left(1-\frac{c}{2 f l}\right)^{2}}} .
$$

Eq. (31) and (42) can now be combined to derive a closed form equation for the long cable $L \ll l$ configuration

$$
\left.E_{\theta}^{\max }(f)\right|_{L \ll l}= \begin{cases}\frac{60 I_{m}(f)}{r}\left(2 \sin ^{2}\left(\frac{\pi f l}{c}\right)\right), & \text { if } f \leq \frac{c}{2 l} \\ \frac{60 I_{m}(f)}{r} \frac{2}{\sqrt{1-\left(1-\frac{c}{2 f l}\right)^{2}}}, & \text { if } f>\frac{c}{2 l}\end{cases}
$$

Eq. (43) represents an envelope approximation of Eq. (28). A comparison of the approximation equation and the exact solution are presented in Fig. 12, and it is apparent that the approximation corresponds to the actual characteristics with good accuracy. 
Calculated E-field from CM current

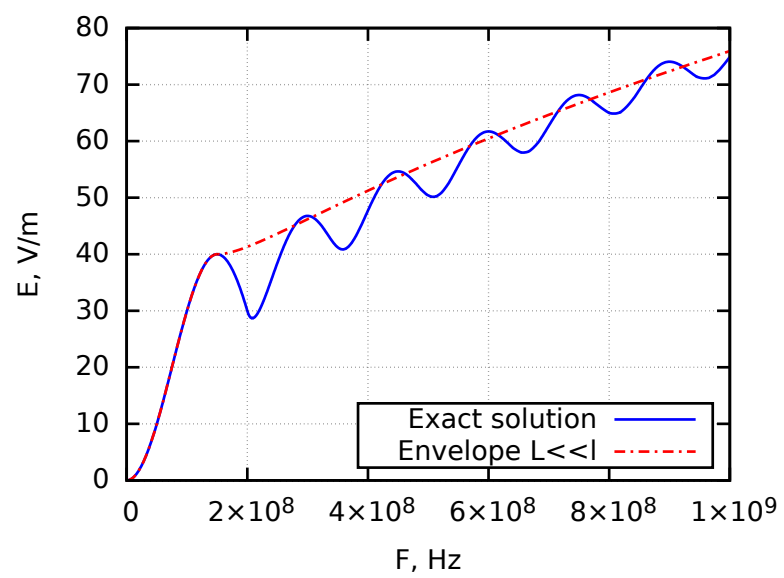

Fig. 12. Comparison of the exact solution (Eq. (15)) and the approximation equation for $L \ll l$ (Eq. (43)). $l=1.0 \mathrm{~m}$, $\boldsymbol{L}=\mathbf{0} \boldsymbol{m}, I_{m}=1 \mathrm{~A}, r=3 m, \phi_{L}=\pi / 2$.

For the approximation equation for the long cable configuration (Section IV), obtaining a transfer coefficient did not depend on the phase $\phi_{L}$ and the reactive load characteristics of the TL. For the approximation equation for the short cable configuration (Section V), it was assumed that the TL load phase $\phi_{L}= \pm \pi / 2$ should be used for the worst case analysis. Fig. 13 illustrates the exact solution for the transfer coefficients for various phases $\phi_{L}$ of the short cable configuration. This figure shows that the maximum transfer coefficient is achieved at $\phi_{L}= \pm \pi / 2$.

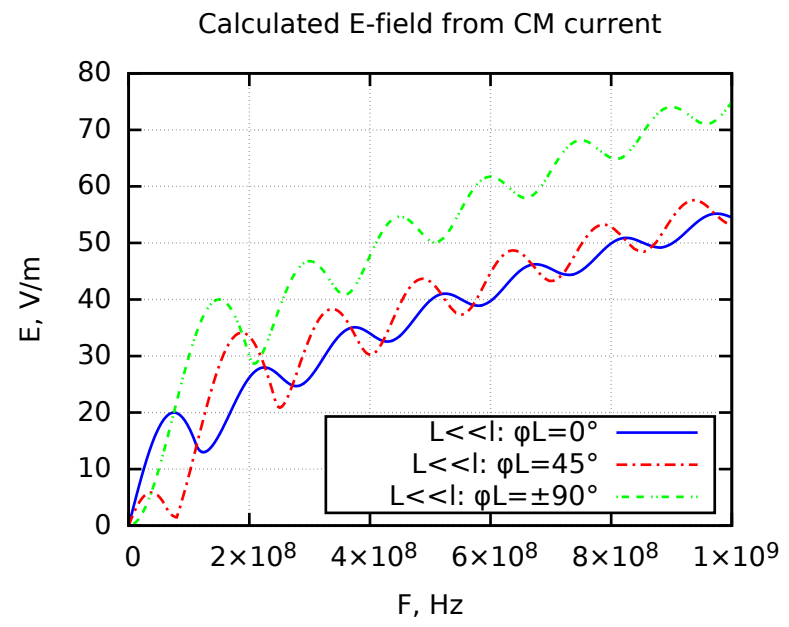

Fig. 13. Comparison of the exact solution for the transfer coefficients from Eq. (15) for various phases $\phi_{L}$ of the $L \ll l$ scenario. $l=1.0 \mathrm{~m}, I_{m}=1 \mathrm{~A}, r=3 \mathrm{~m}$.

Fig. 14 presents a comparison of the coefficients for the approximation equations for the long and short cable configurations. Notably, the long cable approximation envelope should be used for the worst case, as it yields higher transfer values at a low frequency range, making an underestimation less likely in this scenario.

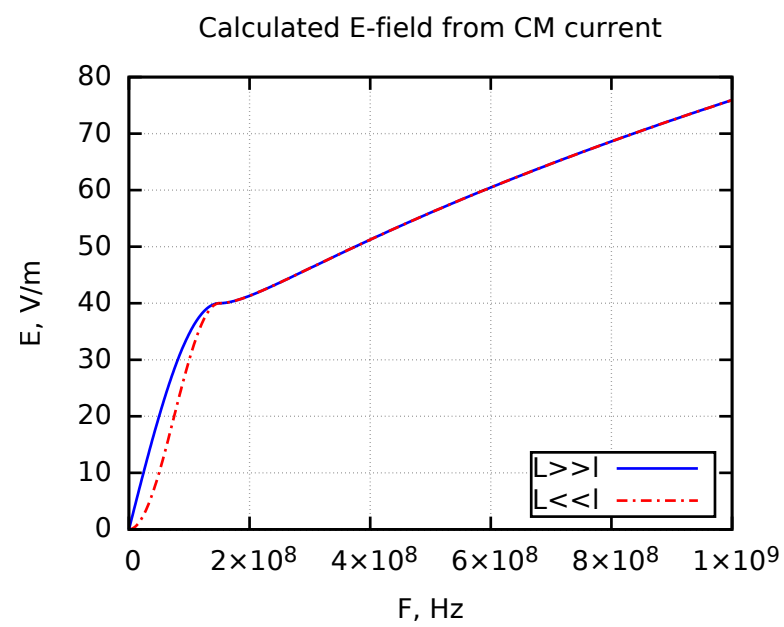

Fig. 14. Comparison of the transfer coefficients from Eq. (26) and from Eq. (43) for the approximation equations for $L \gg l$ and $L \ll l$, respectively. $l=1.0 \mathrm{~m}, I_{m}=1 \mathrm{~A}, r=3 \mathrm{~m}$.

\section{Vi. Calculation Procedure}

At this point, we can summarize the procedure for radiated field prediction via measurement of the CM current in the cable attached to the DUT. The following procedure is proposed for estimating the radiated field:

- The working DUT should be placed on a nonconductive table at a height of $0.8 \mathrm{~m}$ to $1 \mathrm{~m}$ from the conductive metal floor.

- A CM current probe should be connected to a spectrum analyzer that ideally has the same setup parameters as the EMI receiver of the SAC chamber, as per EN 55032 standards. For example, detector type: peak or quasi-peak, and CISPR filter resolution bandwidth (at $-6 \mathrm{~dB}$ ): 120 $\mathrm{kHz}$.

- The CM current probe measurement should be performed along the entire length of the attached cable. As noted in Section IV-A, for the low frequency range $f \leq c /(2 l)$, the measurement of the maximum current magnitude must also be performed along the horizontal portion of the cable to determine the peak magnitude of the CM current. An alternative approach is described in Section IV-B.

- Only a limited number of noise current spurs or peaks at problematic or resonance frequencies $I_{m 1}\left(f_{1}\right), I_{m 2}\left(f_{2}\right) \ldots$ should be registered. It is not necessary to register the position of the current probe on the cable.

- Problematic points $I_{m 1}\left(f_{1}\right), I_{m 2}\left(f_{2}\right) \ldots$ can now be transformed to a radiated emission electric filed using Eq. (26) to derive an approximation of the long cable configuration as the worst case.

Eq. (15) can be used as an exact solution. However, for each frequency, the angle $\theta$ for maximum field strength must be scanned or numerically determined. Another drawback is that the characteristics of Eq. (15) includes many local minima and maxima (Fig. 7). In this case, one of the parameters (such as $L$ or speed of light $c$ ) must be varied to correctly overlap the measured peaks with those calculated using Eq. (15). 
To automate this process of calculating radiated emissions, the script for the Maxima algebraic system software has been developed as an active media for this study.

\section{EXPERIMENT}

\section{A. Equipment and setup}

The following equipment were used for the experimental validation of the model:

- CM current probe: Rohde\&Schwarz EZ-17 model 2, with its maximum frequency specified as $200 \mathrm{MHz}$.

- Spectrum analyzer: Rohde\&Schwarz FPL1003 (equipped with a battery).

- SAC: EN 55032 Class B (3 m), Ektos TRS A/S [13].

- DUT: Advent Technologies A/S SereneU-5 5kW fuel cell unit [14], with three modes of operation: Standby, Bleed, and Conversion. The unit has a metal enclosure. Hence, it can be assumed that most radiation will be from the attached cable.

- In keeping with the setup, the following parameters are used in Eq. (26): $r=3$ as the distance from the DUT to the antenna in the SAC; and $l=1$, which includes a turntable height of $0.8 \mathrm{~m}$ plus the vertical distance to the cable inlet on the DUT; and the speed of light $c=$ $3 \cdot 10^{8} \mathrm{~m} / \mathrm{s}$.

The setup of the DUT in the SAC chamber for a radiated emission test is presented in Fig. 3. The DUT has a power cable for connecting the electronic load and an ethernet cable for controlling the unit. A power cable with two wires having a cross-section of $35 \mathrm{~mm}^{2}$ lies on the floor and exits through the wall of the chamber.

\section{B. Power cable radiation: Conversion mode}

During the power conversion operation by the unit, the CM current was measured along the length of the power cable using a spectrum analyzer to determine the critical frequencies with maximum current. The results from the spectrum analyzer measurements at some points where peaks of $\mathrm{CM}$ current were found are presented in Fig. 15. The maximum CM current was found in the portion of the power cable close to the outlet in the wall of the chamber (Fig. (15f)) due to the noisy electronic load placed outside the chamber. A radiated emission full scan was performed, and the results are presented in Fig. 16.

It is now possible to compare the radiated emissions with that calculated using Eq. (26) for the long cable configuration. For comparison, the $\mathrm{CM}$ current measurement data from Fig. 15f was used because maximum peaks were found there. The input power measurements $(\mathrm{dBm})$ from the spectrum analyzer were converted to voltage values for every frequency (considering $50 \mathrm{Ohm}$ of input impedance) and then to the magnitude of the current $I_{m}$, using the current sensor EZ17 transfer factor. This current value array $I_{m}(f)$ is used in Eq. (26) to calculate the radiated electric field strength. The result of the comparison is presented in Fig. 17.

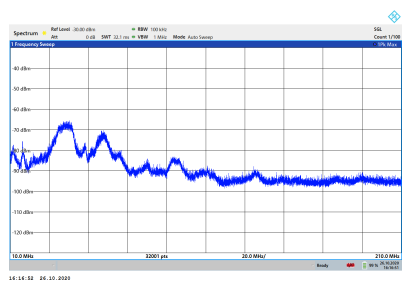

(a)

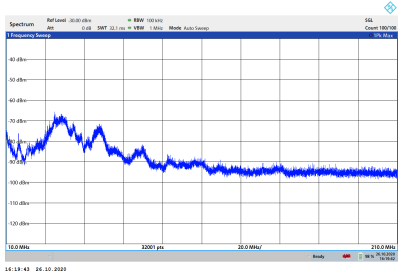

(c)

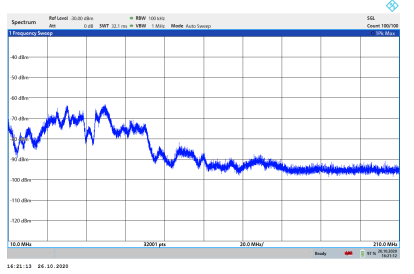

(e)

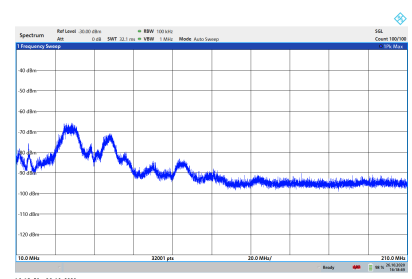

(b)

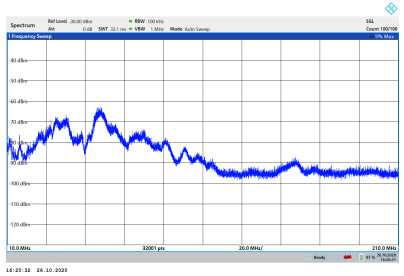

(d)

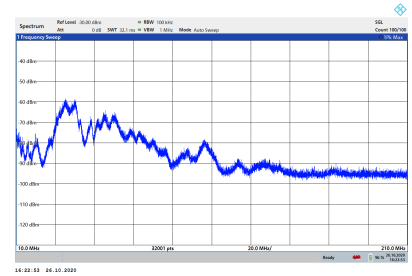

(f)
Fig. 15. Results for $\mathrm{CM}$ current measurement along the entire length of the power cable using s spectrum analyzer. Conversion mode.

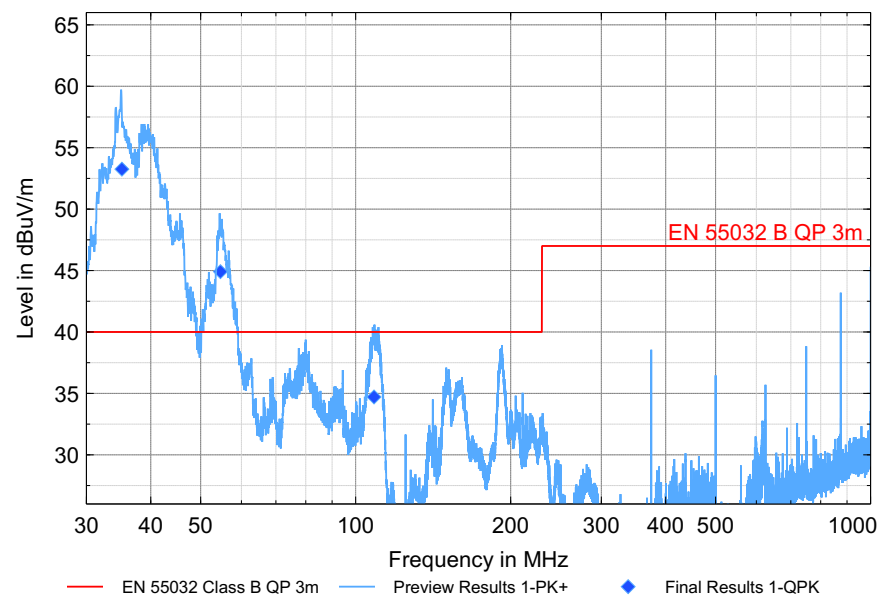

Fig. 16. SAC radiated emission results for power cable radiation in Conversion mode.

\section{Power cable radiation: Bleed mode}

The DUT unit was tested in another mode of operation (Bleed mode). Similarly, the CM current was measured along the entire length of the power cable. The results from the spectrum analyzer at different points of the cable are presented in Fig. 18. The derivation of the maximum current is presented in Fig. 18e.

An SAC radiated emission full scan was performed using 


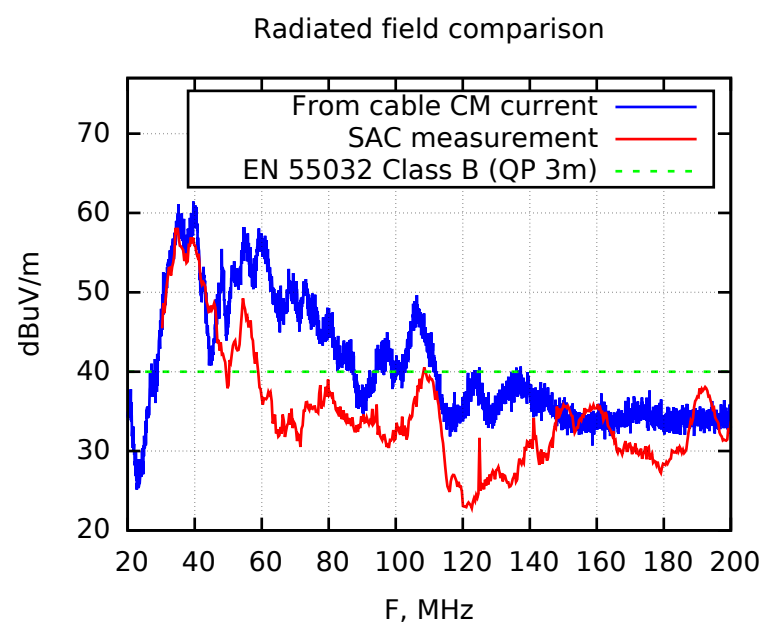

Fig. 17. Comparison of the calculated (Eq. (26)) and SAC measured radiated emission field. The $\mathrm{CM}$ current in the power cable is measured. Spectrum analyzer data was obtained from Fig. (15f).

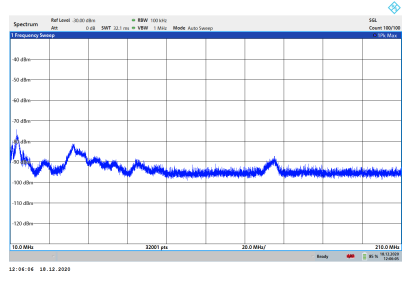

(a)

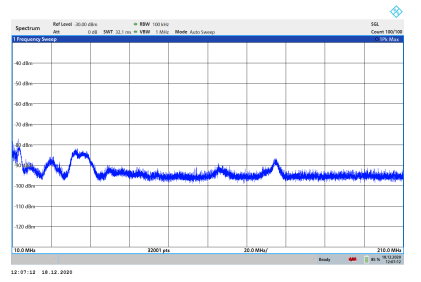

(c)

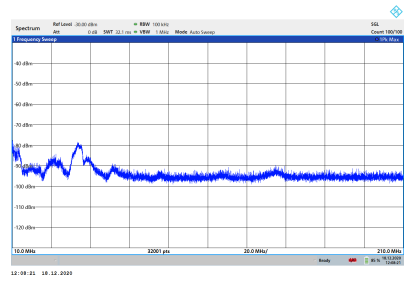

(e)

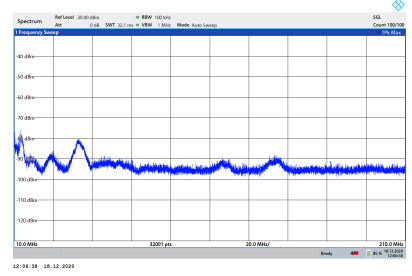

(b)

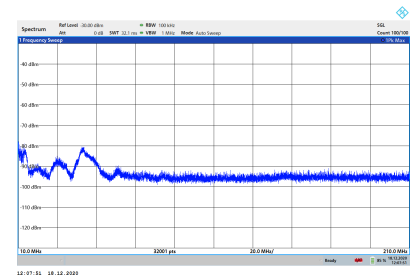

(d)

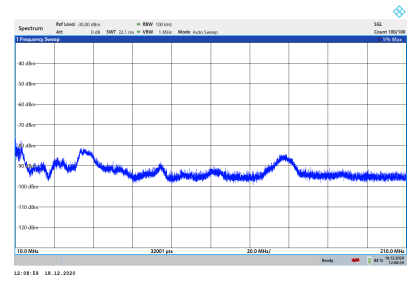

(f)
Fig. 18. Results for the CM current measurement along the entire power cable length using a spectrum analyzer. Bleed mode.

only a peak detector to speed up the process. The results are presented in Fig. 19.

The comparison of the calculated and measured radiated field is presented in Fig. 20.

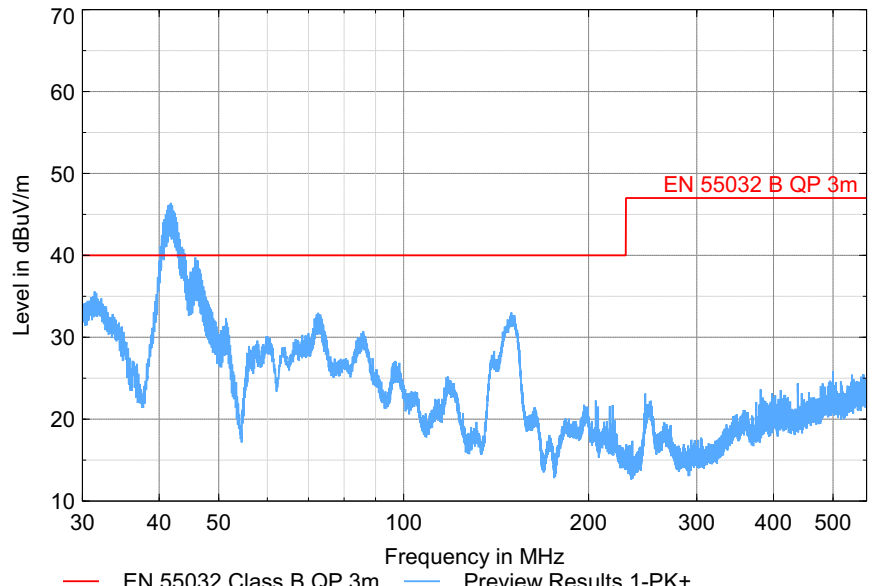

Fig. 19. SAC radiated emission scan results for power cable radiation in Bleed mode.

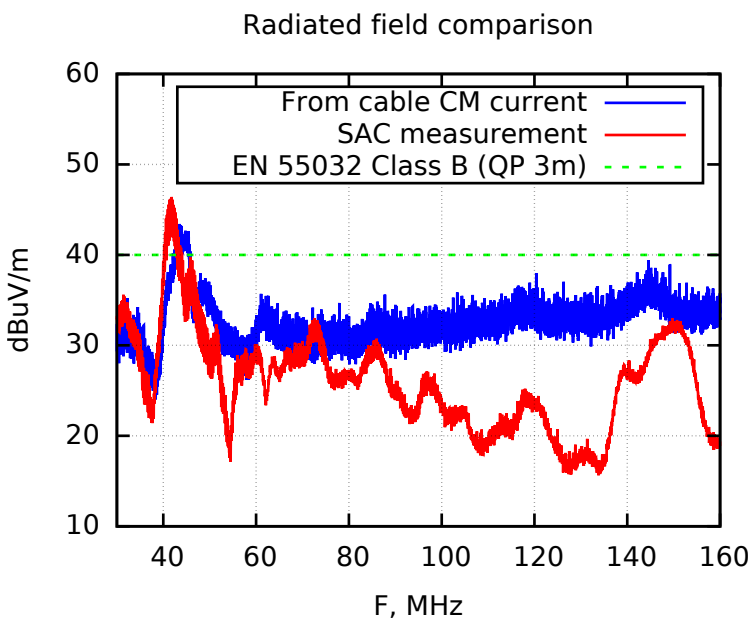

Fig. 20. Comparison of the calculated (Eq. (26)) and SAC measured radiated emission fields. CM current measured in the power cable in Bleed mode. Spectrum analyzer data was obtained from Fig. 18e.

\section{Ethernet cable radiation: Standby mode}

In Standby mode, an ethernet cable was attached to the DUT unit. The spectrum of the CM current in the ethernet cable with maximum values is presented in Fig. 21.

An SAC radiated emission full scan was performed only with a peak detector to speed up the process. The results are presented in Fig. 22.

The comparison of the calculated and measured radiated field is presented in Fig. 23.

Notably, from the comparison of the experimental results (Fig. 17 and Fig. 20) the radiated emission field measurement and prediction are in good agreement at lower frequencies (below $60 \mathrm{MHz}$ ). In the frequency range $50-150 \mathrm{MHz}$, the predicted emission has some overestimation of approximately $10 \mathrm{~dB}$. This overestimation may be attributable to the fact that at these frequencies, the maximum radiation from the vertical portion of the cable can be located at angles $\theta$, which are 


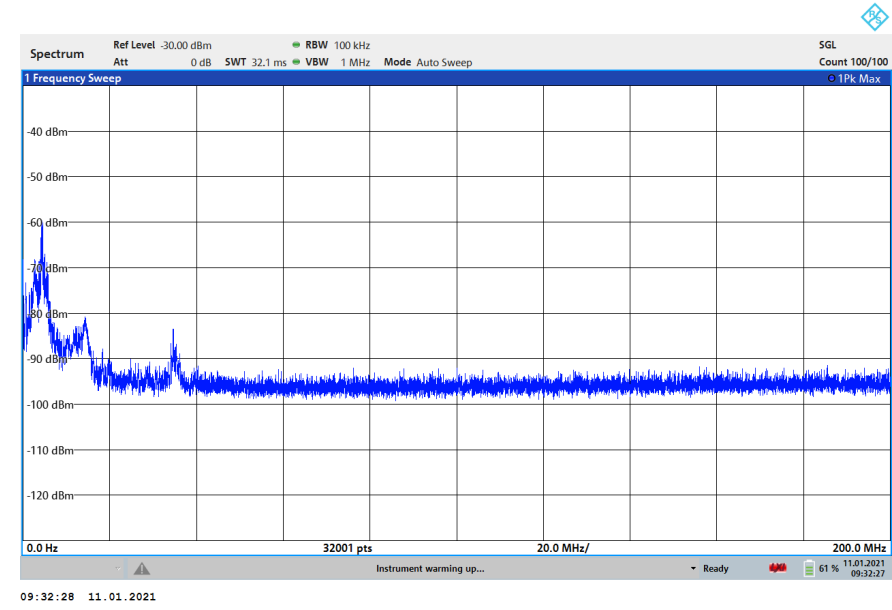

Fig. 21. Results for CM current measurement along the ethernet cable using a spectrum analyzer. Standby mode.

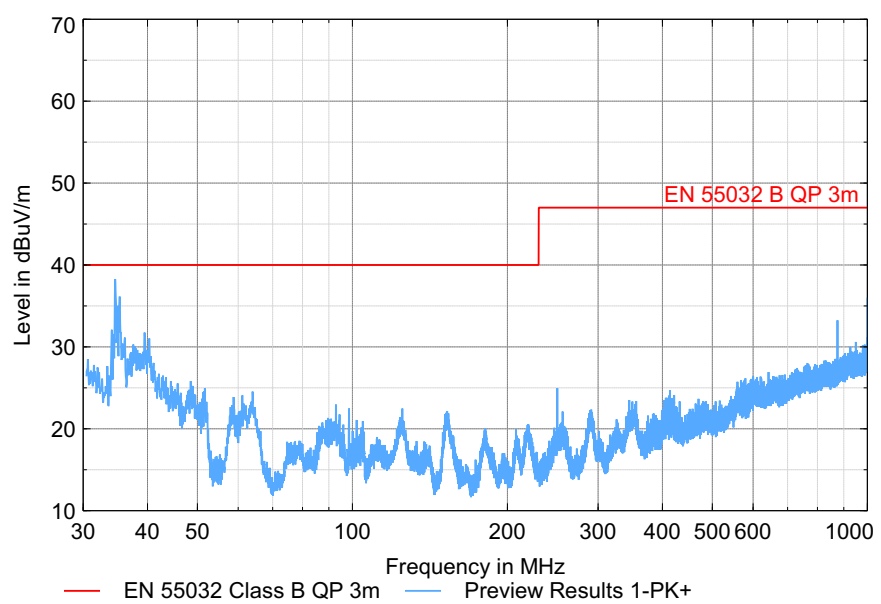

Fig. 22. SAC radiated emission results for ethernet cable radiation in Standby mode.

in a different direction from the antenna in the SAC. The metal case of the DUT can also affect the radiation pattern. Another reason might be that the distance from the antenna to the DUT $r=3 m$ is not large enough for the far zone criteria. Hence, some errors can appear at low frequencies. The overestimation described can be taken into account during an EMC troubleshooting process. From the point of view of the preparation process, it is better to have an overestimation than an underestimation for a higher probability of passing the radiated emission test at an accredited laboratory.

\section{CONCLUSION}

The proposed model is designed for predicting the radiated emissions from power converters and other electronic devices by measuring the $\mathrm{CM}$ current in the attached cable. The maximum measured $\mathrm{CM}$ current peak at the curtain frequency is used to predict the radiated field at that frequency. It is not necessary to register the coordinate position of the point where the maximum CM current was found, as only the magnitude and frequency are significant.

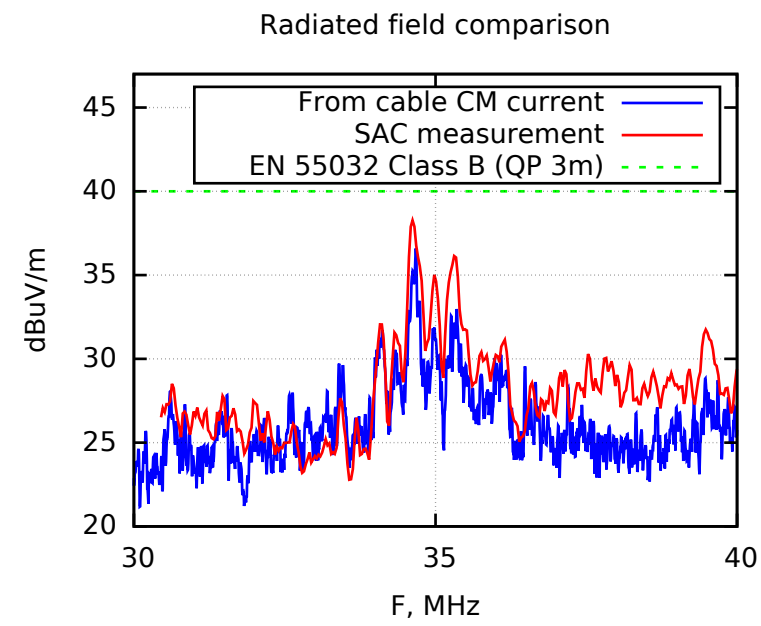

Fig. 23. Comparison of the calculated (Eq. (26)) and SAC measured radiated emission fields. The CM current in the ethernet cable is measured in Standby mode. Spectrum analyzer data was obtained from Fig. 21.

The model is based on a TL loaded with reactive impedance and the assumption that the current distribution along the cable follows a sinusoidal distribution law. The analytic equation of the radiation pattern has been derived, and a simplified approximation equation has been also presented. The model approximation was developed for two configurations. The first approximation is used for a long cable configuration, with the horizontal portion of the cable lying on the floor of the SAC. This configuration is most often used in power electronics in which the cables are thick and cannot be threaded out in a standard way. Another configuration is a short cable, which corresponds to traditional tests in which the cable exits the chamber directly beneath the DUT through the bottom of the turntable. The results of the comparison and analysis show that the approximation of the long cable configuration may be used in all cases as a universal approach, using Eq. (26). It is also shown that the measurement of the CM current peaks or nodes must be performed along the entire length of the cable and not only along the vertical portion.

The proposed model was experimentally validated using a fuel cell converter module at $5 \mathrm{~kW}$ output power. The Maxima software code for automated calculation of the radiated field from the measurement data is supplied as supplemental material.

\section{REFERENCES}

[1] Williams, Tim. EMC for product designers. Newnes, 2016.

[2] Hwan-Woo Shim and T. H. Hubing, "Model for estimating radiated emissions from a printed circuit board with attached cables due to Voltage-driven sources," in IEEE Transactions on Electromagnetic Compatibility, vol. 47, no. 4, pp. 899-907, Nov. 2005, doi: 10.1109/TEMC.2005.859060.

[3] C. Su and T. H. Hubing, "Improvements to a Method for Estimating the Maximum Radiated Emissions From PCBs With Cables," in IEEE Transactions on Electromagnetic Compatibility, vol. 53, no. 4, pp. 10871091, Nov. 2011, doi: 10.1109/TEMC.2011.2165217. 
[4] S. Deng, T. Hubing and D. Beetner, "Estimating Maximum Radiated Emissions From Printed Circuit Boards With an Attached Cable," in IEEE Transactions on Electromagnetic Compatibility, vol. 50, no. 1, pp. 215-218, Feb. 2008, doi: 10.1109/TEMC.2007.915288.

[5] S. Shinde et al., "Radiated EMI Estimation From DC-DC Converters With Attached Cables Based on Terminal Equivalent Circuit Modeling," in IEEE Transactions on Electromagnetic Compatibility, vol. 60, no. 6, pp. 1769-1776, Dec. 2018, doi: 10.1109/TEMC.2017.2782659.

[6] D. Tashiro, T. Hisakado, T. Matsushima and O. Wada, "SingleConductor Transmission Line Model Incorporating Radiation Reaction," in IEEE Transactions on Electromagnetic Compatibility, vol. 63, no. 4, pp. 1065-1077, Aug. 2021, doi: 10.1109/TEMC.2020.3041468.

[7] Mat Aschenberg \& Charles Grasso, Radiation from Common-Mode Currents - Beyond $1 \mathrm{GHz}$ (Three Methods Compared).

[8] Balanis, Constantine A. Antenna theory: analysis and design. John wiley \& sons, 2015

[9] Shyfryn Ya. S. (1976) Antenny [Antennas]. Kharkov, VYRTA ym. L. A. Hovorova Publ., 408 p.

[10] G.N. Kocherzhevsky. Antenna and feeder devices. - Moscow: Svyaz, 1972.

[11] J. Jia, D. Rinas and S. Frei, "Prediction of radiated fields from cable bundles based on current distribution measurements," International Symposium on Electromagnetic Compatibility - EMC EUROPE, 2012, pp. 1-7, doi: 10.1109/EMCEurope.2012.6396759.

[12] G. McCormick, Z. A. Khan, V. Devabhaktuni, M. Alam and A. Wood, "Estimating radiated emissions from printed circuit boards and cables inside EMC chambers," 2010 IEEE International Symposium on Electromagnetic Compatibility, 2010, pp. 36-39, doi: 10.1109/ISEMC.2010.5711243.

[13] https://ektos.net/testing-reliability-services/.

[14] https://www.serenergy.com/units/. 